\title{
Psychosocial treatment approaches to difficult-to-treat depression
}

D

espite adequate first-line treatment for depression, $50 \%-60 \%$ of patients remain symptomatic. ${ }^{1}$ These people require more outpatient visits and psychotropic medications than do those who respond to initial treatment, ${ }^{2,3}$ and they continue to have impaired social and occupational functioning and morbidity from other illness. ${ }^{4}$ This difficult-to-treat depression (DTTD) is therefore a considerable burden in the community, and current management options are only partially effective. ${ }^{5-7}$

There are several approaches to defining DTTD. ${ }^{5,8,9} \mathrm{~A}$ common pragmatic conceptualisation is that of treatmentresistant depression, usually defined as a failure to respond to one or more adequate trials of antidepressants. Emerging themes that may explain the occurrence of DTTD in patients include coexisting psychiatric and medical conditions, environmental and contextual factors, inadequate diagnosis and treatment, medication nonadherence, and issues such as low self-esteem, hopelessness and cognitive reactivity.

Psychosocial interventions are widely used in the treatment of major depressive disorder (MDD) ${ }^{10}$ but are less routinely applied in DTTD, despite some evidence of their efficacy.11,12 A possible reason for this is the dominance of biological treatments in the research literature for DTTD. ${ }^{5,11,13}$ A systematic review of randomised trials using psychotherapy for DTTD identified only six unique studies, all but one of which showed efficacy for psychotherapy in DTTD. ${ }^{12}$ Biological approaches to the management of DTTD are outlined elsewhere in this supplement (see Chan et al, page 44; and Fitzgerald, page 48). ${ }^{8,14}$ Here, we consider the role of psychosocial interventions.

We conducted a review of publications examining psychosocial interventions in DTTD using search phrases including: treatment-resistant depression, refractory depression, treatment-refractory depression, psychosocial, psychology, CBT, therapy, and risk factors. Articles identified included review papers, uncontrolled pilot studies, case reports and randomised controlled trials (RCTs).

\section{Links between psychosocial factors and DTTD}

\section{Medical and psychiatric comorbidity}

Patients with depression, particularly those with chronic depression or DTTD, often have coexisting medical and psychiatric conditions. ${ }^{7,15,16}$ In patients with comorbid depression and medical conditions (eg, thyroid disorders, infections, neurological conditions), the depression is often untreated or unrecognised because of shared symptoms. ${ }^{7}$ The United States National Comorbidity Survey found most lifetime cases of depression were secondary to another psychiatric disorder and that $75 \%$ of people with depression had another psychiatric disorder. ${ }^{17}$ Anxiety and depression often coexist, so that patients with a diagnosis

\section{Summary}

Coexisting psychiatric and medical conditions, environmental and contextual factors, inadequate diagnosis and treatment, medication non-adherence, and issues such as low self-esteem, hopelessness and cognitive reactivity, can play a role in difficult-to-treat depression.

- A reduction in symptoms due to pharmacological treatment does not equate with full recovery, and some level of rehabilitation is often required.

- The evidence base for psychosocial therapies in difficult-to-treat depression is small, with the research heavily weighted toward biological treatments. Nevertheless, combining psychological treatments with pharmacotherapy is likely to improve rates of recovery in difficult-to-treat depression.

- Psychological therapies can be useful in modifying health beliefs, treating comorbid anxiety and other disorders, dealing with contextual factors, and activating patients to facilitate their recovery.

- Effective treatment requires a multidisciplinary team involving a general practitioner and psychologist, and sometimes a psychiatrist. Effective communication and active engagement of patients and families is essential.

of depression commonly experience an anxiety disorder, such as generalised anxiety disorder, obsessivecompulsive disorder or panic disorder (see Tiller, page 28). ${ }^{18,19}$ Alcohol and other substance dependencies and personality disorders also frequently coexist with depression, ${ }^{1,18}$ and recent studies have begun to identify "hidden" bipolarity features as significant factors for treatment resistance. ${ }^{20}$ Complexity and comorbidity should be considered the norm. ${ }^{7,15}$ These comorbidities complicate the clinical picture, worsen the depression, and make it difficult to treat.

\section{Environmental and contextual factors}

Patients with a diagnosis of depression, and specifically DTTD, often experience a range of negative environmental or contextual factors, such as family conflict, financial distress, work stress or job loss, poor-quality interpersonal relationships and social support, poor social adjustment, and other serious life events. ${ }^{1,4,5,15,21}$ Adverse health behaviour such as obesity, sedentary lifestyle and smoking are also identified risks for people with MDD. ${ }^{22}$ A recent systematic review of 25 primary studies involving 5192 patients with chronic depression identified that negative social interaction and low levels of social integration coexist with chronic depression. ${ }^{16}$ There is consensus that late onset of depression, family history of DTTD, and severity or chronicity of depression are also risk factors for treatment resistance. ${ }^{15,18}$ 


\section{Inadequate diagnosis and treatment}

Depression in primary care appears in many guises. Twothirds of patients with depression initially present with one or more physical symptoms, ${ }^{23}$ and comorbid anxiety, personality traits, addictive behaviour and bipolarity can all potentially alter the clinical presentation. Furthermore, although there are probably different types of depression, such as melancholic, hopeless or situational depression, ${ }^{24}$ these heterogeneous presentations are often treated as though they are the one condition.

If a patient has not responded fully to the first-line treatment, it is always necessary to review the diagnosis and the treatment. Antidepressant medication and psychotherapy are both recommended as first-line treatments, either alone or in combination, for patients with mild to moderate MDD. ${ }^{25}$ However, these treatments are not always equally efficacious. For example, there is evidence that depression characterised by severe hopelessness responds poorly to antidepressants. ${ }^{26}$ Coexisting psychiatric disorders and medical illnesses require treatment in their own right if the treatment for depression is to be successful. ${ }^{16}$

Similarly, persisting contextual factors need to be taken into account when making a comprehensive diagnosis of the situation. Continuing stressors, or poor resources and coping skills, have a ceiling effect on the level of improvement that can be expected from pharmacotherapy alone. $^{2,6}$

\section{Medication non-adherence}

Patients with depression often do not take, forget to take, or cannot be bothered to take their medication. ${ }^{18}$ This non-adherence to antidepressants is explained partly by personality characteristics, ${ }^{27}$ the strength of the doctorpatient alliance, ${ }^{28}$ intolerable side effects, ${ }^{18}$ and health beliefs and stigma. ${ }^{29}$ Patients' attitudes and beliefs are at least as important as side effects in predicting adherence to medication. ${ }^{29}$ Furthermore, the depression, anhedonia and secondary demoralisation (the "giving up", "can't be bothered", "it won't help" attitude) that so often accompany depressive illness reinforce the likelihood of non-compliance.

\section{Low self-esteem, hopelessness, anhedonia and cognitive reactivity}

Individuals with chronic depression that is difficult to treat tend to experience significant low self-esteem, anxiety, demoralisation, hopelessness, and lack of joy and drive (anhedonia). ${ }^{21,30}$ In some people, these may be lifelong traits; in others, they may be activated by the depression. ${ }^{30}$ Nevertheless, people with treatment-resistant depression have more negative affect and dysfunctional cognitions than those with non-resistant depression, ${ }^{31}$ and people who experience repetitive and unhelpful thinking about depression tend to have prolonged depressive symptoms. ${ }^{30,32}$ Severe and persistent depression occurs when there is a recurring reinforcing relationship between depressed mood and negative cognitive processing. This phenomenon, in which the symptoms of depression and secondary beliefs maintain each other, producing a continuous cycle of reinforcement, is described as cognitive reactivity or cognitive vulnerability. ${ }^{30,32}$

\section{Usefulness of psychological interventions}

Patients' thinking and behaviour play a large role in determining outcomes of treatment for depression ${ }^{32}$ and are thus prime candidates for intervention through a psychosocial treatment regimen. Evidence-based psychological therapies can be used to explore and overcome interpersonal difficulties, health beliefs and stigma, medication non-adherence, anhedonia and ruminative thinking, and can assist with the behavioural changes necessary to encourage patient activation and full recovery.

Psychological therapies with Level I evidence for use in depression are cognitive behaviour therapy (CBT), interpersonal psychotherapy (IPT), family-based therapy (FBT) and brief psychodynamic psychotherapy (BPP). Mindfulness-based cognitive therapy (MBCT) has Level III evidence for depression. ${ }^{10}$ All these therapies are brief and manualised, which allows them to be applied in a reliable way, such that their efficacy can be examined in research trials.

While considerable research has demonstrated the effectiveness of psychotherapeutic interventions in MDD, there has been substantially less investigation of these in DTTD. ${ }^{5,12}$ A recent critique of current novel antidepressant and brain stimulation therapies for treatment-resistant depression contended that psychotherapy substantially increases response rates in non-psychotic depression, and that the restrictive definition of treatment-resistant depression should be expanded to include failure to respond to psychotherapy. ${ }^{33}$ Several other case studies, reviews, uncontrolled pilot studies, open studies and RCTs provide evidence for the use of psychotherapy in DTTD. $6,31,32,34-36$ The range of potential psychosocial treatments for DTTD is shown in the Box.

A patient-centred approach involving adequate education, assessing a patient's motivation and readiness for treatment, preparing the patient to accept treatment, and communicating realistic treatment expectations is an important clinical skill that has been shown to facilitate medication adherence. ${ }^{37} \mathrm{CBT}$ and regular contact with the treating clinician may encourage medication adherence during treatment of depression. ${ }^{13}$

\section{Cognitive behaviour therapy}

A recent systematic review of 13 articles, representing seven RCTs with a total of 592 patients, concluded that psychotherapy had utility in managing treatment-resistant depression. ${ }^{12}$ Six of the trials used cognitive therapy. CBT was mostly used to augment antidepressants, but in two trials it was a standalone treatment, delivered in 16 sessions. The review concluded that primary care providers should consider psychotherapy as a reasonable treatment option for treatment-resistant depression. ${ }^{12}$ In another study, improvements in social functioning and reductions in depressive symptoms were observed after group CBT, and were maintained at 12 -month follow-up. ${ }^{11} \mathrm{~A}$ combination of CBT and medication was found to be effective in patients with chronic depression and those with residual symptoms. ${ }^{38}$

CBT techniques are useful in dealing with some of the specific risk factors associated with DTTD. ${ }^{12,16,21,35}$ For 


\section{Factors associated with difficult-to-treat depression and psychological} interventions that can help

Medical and psychiatric comorbidity (cancer; endocrine, heart or inflammatory disease; substance dependency; anxiety disorders; personality disorders; bipolarity):

- Clarification and differentiation of comorbid psychiatric conditions, including contribution of personality

- Specific treatment of anxiety disorders and addictive behaviour

Environmental and contextual factors (family and relational conflict; social and interpersonal problems; financial distress; work stress or job loss; weak social supports; serious illness; other serious life event):

CBT: behavioural interventions and problem-solving techniques can be useful in developing social skills and resolving interpersonal difficulties

IPT: can address social and interpersonal issues, work stress, job loss and adjustment to life events or change. Helps patient understand how these problems lead to becoming distressed and depressed and facilitates making appropriate changes or adjustments

FBT: to address the manner in which family members interact, so as to improve family relationships and communication, reduce stress and increase support BPP: to explore and work through conflicts within oneself and with others that affect relating and coping styles (eg, avoidance or denial, need for approval). Draws on the patient's accounts of current and past relationships, and the relationship with the therapist (transference)

Inadequate diagnosis and treatment (comorbidities; types of depression

[melancholic or anhedonic, demoralisation or hopelessness, situational reaction];

unresolved environmental influences):

- Review of psychiatric, social and psychological aspects of diagnosis

- Specific treatments for comorbidities (eg, CBT for anxiety disorders)

- Cognitive therapy for hopeless depression; remoralisation therapy for demoralisation

Problem solving for unresolved social stressors

\section{Medication non-adherence:}

Psychoeducation

Review of health beliefs, stigma and attitudes to depression and medication

Support of motivation for recovery

\section{Low self-esteem, hopelessness, anhedonia and cognitive reactivity:}

- CBT: to help combat hopelessness, other negative thoughts and unhelpful core beliefs associated with low self-esteem. Scheduling of pleasant events can (paradoxically) be effective in anhedonia. Exposure and relaxation (eg, deep breathing exercises) reduce anxiety. Motivational interviewing helps motivation and patient activation. Activity scheduling can help increase activity level, which increases physical exercise and hedonic activity

- MBCT: to facilitate relaxation and, through encouraging acceptance, help reduce avoidance behaviour, give patient a different attitude and "relationship" to depressive thoughts, and decrease rumination

CBT = cognitive behaviour therapy. IPT = interpersonal psychotherapy. FBT = family-based therapy. $\mathrm{BPP}=$ brief psychodynamic psychotherapy. $\mathrm{MBCT}$ = mindfulness-based cognitive therapy.

example, the maladaptive cognitions and behaviour that perpetuate chronic depressive symptoms can be modified by CBT techniques such as cognitive restructuring. ${ }^{12,21,35}$ Activity scheduling, social skills training and other behavioural interventions can help overcome anhedonia, interpersonal or social problems, and coexisting anxiety. ${ }^{12,21,36}$

\section{Interpersonal psychotherapy}

There is considerable evidence that IPT is effective in treating depression, particularly around improving the quality of social relationships and interpersonal skills. ${ }^{10}$ There is little research about the effectiveness of IPT in DTTD, but it has been recommended for the treatment of chronic depression. ${ }^{39}$ IPT, as an additional or standalone treatment, has been found to be beneficial in people with recurrent MDD. ${ }^{40}$ Features of IPT that make it potentially beneficial in treating people with DTTD or chronic depression include: attention to the therapeutic alliance and the patient's readiness to change; the case formulation that considers issues of attachment, culture, self-definition and interpersonal relatedness; and its addressing of effects of trauma, abuse or insecure attachment on patterns of relating and interpersonal sensitivity. ${ }^{41}$ IPT can also help deal with aspects of grief, relational conflict and role transition that may be intrinsic parts of the depression or consequences of it.

\section{Family-based therapy}

FBT is designed to improve communication and resolve conflicts between family members. There is considerable evidence that family therapy is effective in treating depression, particularly as individuals with depression report significant problems in multiple areas of family functioning. ${ }^{10}$ However, there is little research evidence regarding its efficacy in DTTD. Family members of patients with depression experience change in their own lives because of the depression. ${ }^{2}$ The way in which family members respond to the depression can have a significant effect on whether the patient will engage in treatment, as well as the duration of the depressive episode. ${ }^{2}$ There is a need for research to identify the precise mechanisms of change activated through FBT. A meta-analysis of research conducted in a related area - family therapy as an adjunct treatment for chronic disease interventions - showed it was efficacious, but which components of the therapy led to the change are still not understood. ${ }^{2}$ Nevertheless, outcome benefits of FBT have been found after five sessions. $^{2}$

\section{Brief psychodynamic psychotherapy}

There is considerable evidence that BPP is effective in treating depression, particularly in helping patients overcome problems in regulating complex feelings, ${ }^{10}$ but there is little research about its effectiveness in DTTD. In a small sample of 10 patients with treatment-resistant depression who were provided with about 12 sessions of BPP, substantial functional and symptomatic gains were associated with the therapy. ${ }^{35}$

\section{Mindfulness-based cognitive therapy}

Studies have shown that MBCT, which combines CBT with mindfulness techniques, is effective in reducing relapse rates in people with multiple episodes of depression. ${ }^{42}$ MBCT has also been shown in pilot studies to be useful in people with DTTD. ${ }^{31,32,36}$ A single RCT showed MBCT to be effective in chronic depression. ${ }^{43}$ MBCT is useful in decreasing ruminative thinking, as well as in changing the relationship the patient has with his or her unhelpful depressive thoughts and feelings, anhedonia, low selfesteem and feelings of hopelessness. ${ }^{31}$

\section{Conclusions}

The goal of treatment for patients with depression is remission of symptoms, coupled with a return to a state of functioning and wellbeing. In clinical practice, antidepressant medications are frequently used, as they seem at face value to be more cost-effective than a course of psychotherapy. However, there is strong evidence to support the use of psychological treatments in depression 
in general, and a smaller amount of evidence to support its use in DTTD and chronic depression.

It is clear that DTTD is often accompanied or complicated by a range of primary or secondary psychological and interpersonal issues that may benefit from attention through non-pharmacological techniques. Before deeming a patient's DTTD to be "treatmentresistant", a thorough assessment should be made to determine whether the depressive symptoms are being complicated by coexisting medical, psychiatric, personality, environmental or social factors. It is also crucial that patients are educated about their depressive illness and the potential contributing factors. The importance of medication adherence needs to be emphasised, and the patient should be supported through regular contact with the treating clinician. Specific psychological therapies should be offered to assist with dysfunctional thinking (depressive thoughts and ruminations) and behaviour (non-compliance, addictions), comorbid anxiety, social integration (to increase social support), and rehabilitation (to engage in productive work). If the patient is ambivalent about accepting treatment, an exploration of health beliefs and motivation through therapy would be useful.

The effective treatment of a person with depression is a multidisciplinary concern involving a doctor and a psychologist, and sometimes a psychiatrist. Communication among health professionals is important, as is engagement with the patient's family, to ensure there is a comprehensive and integrated plan of recovery. Combining pharmacotherapy with a range of cognitive, behavioural and interpersonal strategies is seemingly beneficial. However, there remains a need for more well conducted quality trials investigating effectiveness of psychosocial interventions in DTTD.

Competing interests: No relevant disclosures.

Provenance: Commissioned by supplement editors; externally peer reviewed.

1 Fava M. Diagnosis and definition of treatment-resistant depression. Biol Psychiatry 2003; 53: 649-659.

2 Keitner GI, Mansfield AK. Management of treatment-resistant depression. Psychiatr Clin North Am 2012; 35: 249-265.

3 Crown WH, Finkelstein S, Berndt ER, et al. The impact of treatmentresistant depression on health care utilization and costs. J Clin Psychiatry 2002; 63: 963-971.

4 Thase ME. Treatment-resistant depression: prevalence, risk factors, and treatment strategies. J Clin Psychiatry 2011; 72: el8.

5 Jenkins E, Goldner EM. Approaches to understanding and addressing treatment-resistant depression: a scoping review. Depress Res Treat Epub 2012; Apr 18. doi: 10.1155/2012/469680.

6 Shelton RC, Osuntokun O, Heinloth AN, Corya SA. Therapeutic options for treatment-resistant depression. CNS Drugs 2010; 24: 131-161.

7 Trivedi MH, Daly EJ. Treatment strategies and tactics for treatmentresistant depression. US Psychiatry 2007; 17-20. http://www. touchbriefings.com/pdf/2592/trivedi.pdf (accessed Sep 2012).

8 Chan HN, Mitchell PB, Loo CKA, Harvey SB. Pharmacological treatment approaches to difficult-to-treat depression. MJA Open 2012; 1 Suppl 4 : 44-47.

9 Berlim MT, Turecki G. Definition, assessment, and staging of treatment-resistant refractory major depression: a review of current concepts and methods. Can J Psychiatry 2007; 52: 46-54.

10 Australian Psychological Society. Evidence-based psychological interventions in the treatment of mental disorders: a literature review. 3rd ed. Melbourne: APS, 2010

11 Matsunaga M, Okamoto Y, Suzuki S, et al. Psychosocial functioning in patients with treatment-resistant depression after group cognitive behavioral therapy. BMC Psychiatry 2010; 10: 22.

12 Trivedi RB, Nieuwsma JA, Williams JW Jr. Examination of the utility of psychotherapy for patients with treatment resistant depression: a systematic review. J Gen Intern Med 2011; 26: 643-650.
13 Little A. Treatment-resistant depression. Am Fam Physician 2009; 80: 167-172.

14 Fitzgerald PB. Non-pharmacological biological treatment approaches to difficult-to-treat depression. MJA Open 2012; 1 Suppl 4: 48-51.

15 Maalouf FT, Atwi M, Brent DA. Treatment-resistant depression in adolescents: review and updates on clinical management. Depress Anxiety 2011; 28: 946-954

16 Hölzel L, Härter M, Reese C, Kriston L. Risk factors for chronic depression - a systematic review. J Affect Disord 2011; 129: 1-13.

17 Kessler RC, Nelson CB, McGonagle KA, et al. Comorbidity of DSM-III-R major depressive disorder in the general population: results from the US National Comorbidity Survey. Brit J Psychiatry Suppl 1996; (30): 17-30.

18 Kornstein SG, Schneider RK. Clinical features of treatment-resistant depression. J Clin Psychiatry 2001; 62 Suppl 16: 18-25.

19 Tiller JWG. Depression and anxiety. MJA Open 2012; 1 Suppl 4: 28-31.

20 Dudek D, Rybakowski JK, Siwek M, et al. Risk factors of treatment resistance in major depression: association with bipolarity. J Affect Disord 2010; 126: 268-271.

21 Moore RG, Garland A. Cognitive therapy for chronic and persistent depression. Chichester, UK: John Wiley and Sons, 2003.

22 Katon WJ, Lin EHB, Russo J, et al. Cardiac risk factors in patients with diabetes mellitus and major depression. J Gen Intern Med 2004; 19: 1192-1199.

23 Katon WJ. The many faces of depression in primary care. J Gen Intern Med 2004; 19: 893-895.

24 Clarke DM, Cook K, Smith GC, Piterman L. What do general practitioners think depression is? A taxonomy of distress and depression for general practice. Med J Aust 2008; 188 (12 Suppl): Sll0-S113.

25 American Psychiatric Association. Practice guideline for the treatment of patients with major depressive disorder. Am J Psychiatry 2010; 167 Suppl: A34.

26 Papakostas Gl, Petersen T, Homberger $\mathrm{CH}$, et al. Hopelessness as a predictor of non-response to fluoxetine in major depressive disorder. Ann Clin Psychiatry 2007; 19: 5-8.

27 Cohen NL, Ross EC, Bagby RM, et al. The 5-factor model of personality and antidepressant medication compliance. Can J Psychiatry 2004; 49 106-113.

$28 \mathrm{Lin} E \mathrm{H}$, Von Korff M, Katon W, et al. The role of the primary care physician in patients' adherence to antidepressant therapy. Med Care 1995; 33: 67-74

29 Lingam R, Scott J. Treatment non-adherence in affective disorders. Acta Psychiatr Scand 2002; 105: 164-172.

30 Teasdale JD. Cognitive vulnerability to persistent depression. Cogn Emot 1988; 2: 247-274

31 Eisendrath S, Chartier M, McLane M. Adapting mindfulness-based cognitive therapy for treatment-resistant depression: a clinical case study. Cogn Behav Pract 2011; 18: 362-370.

32 Kenny MA, Williams JM. Treatment-resistant depressed patients show a good response to mindfulness-based cognitive therapy. Behav Res Ther 2007; 45: 617-625

33 Wijeratne C, Sachdev P. Treatment-resistant depression: critique of current approaches. Aust N Z J Psychiatry 2008; 42: 751-762.

34 Abbass AA. Intensive short-term dynamic psychotherapy of treatment-resistant depression: a pilot study. Depress Anxiety 2006; 23: $449-452$

35 Bannan N. Multimodal therapy of treatment resistant depression: a study and analysis. Int J Psychiatry Med 2005; 35: 27-39.

36 Eisendrath SJ, Delucchi K, Bitner R, et al. Mindfulness-based cognitive therapy for treatment-resistant depression: a pilot study. Psychother Psychosom 2008; 77: 319-320.

37 Pampallona S, Bollini P, Tibaldi G, et al. Patient adherence in the treatment of depression. Br J Psychiatry 2002; 180: 104-109.

38 Scott J, Palmer S, Paykel E, et al. Use of cognitive therapy for relapse prevention in chronic depression. Br J Psychiatry 2003; 182: 221-227.

39 Schramm E, Schneider D, Zobel I, et al. Efficacy of interpersonal psychotherapy plus pharmacotherapy in chronically depressed inpatients. J Affect Disord 2008; 109: 65-73.

40 Reynolds CF 3rd, Frank E, Perel JM, et al. Nortriptyline and interpersonal psychotherapy as maintenance therapies for recurrent major depression: a randomized controlled trial in patients older than 59 years. JAMA 1999; 281: 39-45

41 Ravitz P, McBride C, Maunder R. Failures in interpersonal psychotherapy (IPT): factors related to treatment resistances. J Clin Psychol 2011; 67: 1129-1139.

$42 \mathrm{Ma} \mathrm{SH}$, Teasdale JD. Mindfulness-based cognitive therapy for depression: replication and exploration of differential relapse prevention effects. J Consult Clin Psychol 2004; 72: 31-40.

43 Barnhofer T, Crane C, Hargus E, et al. Mindfulness-based cognitive therapy as a treatment for chronic depression: a preliminary study. Behav Res Ther 2009; 47: 366-373. 